\title{
THE PROBLEM WITH PRESENT PROGRESSIVES
}

if you for a moment think you are sacred it is probably just the wheels of the axis creaking which is difficult yes but you withstand it because you must get over that lie and doing so forces a bit more sincerity which might just outlast small talk turn of the world toward evening or morning interesting concepts when you locate them in the past tense and say instead eve or morn likewise acceptable yet I get this feel that all words end with ing are impositions and I'm made to suspect time as the greatest transgressor and to be out of it is to be and to die without complication

do you think the sting of the bee gives it permanence

you recover but you know something has passed you cannot quite place it beneath all words a dissolution a wound that damns it all since the beginning when a bite or a bang or some other contention claimed the language I must disengage from as it stutters when confronted by a stone or a snake or just that gap that must be filled with moments cohered from research or harvest which demands toil

to refuse awareness is to never question is to lose touch is to not remember is to never speak is to die

do you really think the sting of the bee gives it permanence

do y u real y $\mathrm{t}$ ink $\mathrm{t}$ e sti $\mathrm{g} \mathrm{ft} \mathrm{e} b$ e g ves $\mathrm{t} \mathrm{p}$ rm nce

d y $r$ llyt nkt st $\mathrm{ft} \quad \mathrm{b}$ g v s tprm nc 